\title{
Clinical characteristics and temporal profile of recovery in patients with favorable outcomes at 6 months after severe traumatic brain injury
}

\author{
Aditya Vedantam, MD, Claudia S. Robertson, MD, and Shankar P. Gopinath, MD \\ Department of Neurosurgery, Baylor College of Medicine, Houston, Texas
}

OBJECTIVE Early withdrawal of life-sustaining treatment due to expected poor prognosis is responsible for the majority of in-house deaths in severe traumatic brain injury (TBI). With increased focus on the decision and timing of withdrawal of care in patients with severe TBI, data on early neurological recovery in patients with a favorable outcome is needed to guide physicians and families.

METHODS The authors reviewed prospectively collected data obtained in 1241 patients with head injury who were treated between 1986 and 2012. Patients with severe TBI, motor Glasgow Coma Scale (mGCS) score < 6 on admission, and those who had favorable outcomes (Glasgow Outcome Scale [GOS] score of 4 or 5 , indicating moderate disability or good recovery) at 6 months were selected. Baseline demographic, clinical, and imaging data were analyzed. The time from injury to the first record of following commands (mGCS score of 6 ) after injury was recorded. The temporal profile of GOS scores from discharge to 6 months after the injury was also assessed.

RESULTS The authors studied 218 patients (183 male and 35 female) with a mean age of $28.9 \pm 11.2$ years. The majority of patients were able to follow commands (mGCS score of 6 ) within the 1st week after injury (71.4\%), with the highest percentage of patients in this group recovering on Day $1(28.6 \%)$. Recovery to the point of following commands beyond 2 weeks after the injury was seen in $14.8 \%$ of patients, who experienced significantly longer durations of intracranial pressure monitoring $(p=0.001)$ and neuromuscular blockade $(p<0.001)$. In comparison with patients with moderate disability, patients with good recovery had a higher initial GCS score $(p=0.01)$, lower incidence of anisocoria at admission ( $p$ $=0.048)$, and a shorter ICU stay $(p<0.001)$ and total hospital stay $(p<0.001)$. There was considerable improvement in GOS scores from discharge to follow-up at 6 months.

CONCLUSIONS Up to $15 \%$ of patients with a favorable outcome after severe TBI may begin to follow commands beyond 2 weeks after the injury. These data caution against early withdrawal of life-sustaining treatment in patients with severe TBI.

https://thejns.org/doi/abs/10.3171/2017.3.JNS162720

KEY WORDS severe traumatic brain injury; motor recovery; Glasgow Outcome Scale; withdrawal of life-sustaining treatment

$\mathrm{O}$ UTCOMES after severe traumatic brain injury (TBI) are variable, and several studies have focused on predicting clinical outcomes after the injury. ${ }^{3,5,7,}$ 12,15 Many studies have described factors associated with mortality and morbidity after severe TBI. ${ }^{8,12,14,15}$ However, there are few descriptions of the clinical characteristics of patients with favorable outcomes after severe TBI.,4

The temporal profile of early recovery after severe TBI has not been examined in detail. It is often difficult to pre- dict when a patient will show clinical improvement after admission, or if he or she has the potential for a good recovery. In fact, early withdrawal of life-sustaining treatment due to poor prognosis is responsible for the majority of in-house deaths in severe TBI. ${ }^{13,16} \mathrm{With}$ increased focus on the decision and timing of withdrawal of care in patients with severe TBI, ${ }^{16}$ more data on early neurological recovery are needed to inform and guide physicians and families. 
The aim of the present study was to determine the clinical characteristics and temporal profile of recovery in patients with favorable outcomes at 6 months after severe TBI

\section{Methods}

We reviewed a research database containing clinical data on 1241 patients with head injury who were enrolled in prospective TBI studies from 1986 to 2012 . We included patients with severe TBI whose age was $\geq 15$ years; motor Glasgow Coma Scale (mGCS) score $<6$ (i.e., at or deteriorated to $<6$ soon after admission); non-life-threatening systemic trauma (i.e., no organ system with Abbreviated Injury Scale score $>4$ ); and no previous TBI or serious medical problems. Study participants were chosen on the basis of Glasgow Outcome Scale (GOS) scores $(5=$ good recovery, $4=$ moderate disability, $3=$ persistent vegetative state, and 2 = severe disability). Patients with favorable outcomes (GOS score of 4 or 5) at 6 months were selected for this study. All patients were treated in a dedicated Neuro ICU at Ben Taub Hospital according to the individual study protocols, but largely based on the guidelines for treatment of severe TBI at the time.

We reviewed demographic, clinical, and imaging data at admission, as well as duration of intracranial pressure (ICP) monitoring, neuromuscular blockade, and pentobarbital coma. For patients with ICP monitoring, sedation and neuromuscular blockade, if used, were stopped every morning to facilitate a neurological examination. In addition, these patients underwent hourly neurological examinations during the ICU stay. Hourly assessments of GCS scores during the ICU stay as well as results of daily neurological examinations throughout the hospital stay were reviewed. The earliest time to following commands (mGCS score of 6) after severe TBI was noted for each patient in our hospital. The length of stay in the ICU, in the hospital, and the surgical procedures performed during the hospital stay were also recorded. Outcomes were recorded at discharge, 3 months, and 6 months after injury according to the GOS. To further elucidate our practice regarding withdrawal of life-sustaining therapy, we evaluated those patients from our database with severe TBI in whom life-sustaining therapy was withdrawn at our center and compared them to the group of patients with favorable outcomes.

\section{Statistical Analysis}

Descriptive statistics were used for demographic and clinical variables and the values were reported as the mean \pm SD. The chi-square test for categorical variables and the Student t-test for continuous variables were used to identify baseline differences between groups of patients classified by the time to following commands as well as factors associated with good recovery. Fisher's exact test was performed for categorical variables when the number of patients in the subgroup was less than 5. Multiple comparisons for means were performed using the ANOVA test. Pearson's correlation was used to evaluate the correlation between GOS scores at different time points. Statistical analysis was performed using SPSS version 20.0 (IBM), and statistical significance was set at $\mathrm{p}<0.05$.

\section{Results}

Two hundred eighteen patients satisfied the inclusion criteria (Fig. 1). Clinical, demographic, and imaging data are shown in Table 1 . The age range in the study set was 15-70 years. The range of Injury Severity Scores (ISSs) was $16-59$, and the majority of patients $(51.4 \%)$ had an ISS of 25. More than one-third of patients underwent craniotomy or craniectomy at admission, and only 2 patients underwent nonneurosurgical procedures. One patient underwent an exploratory laparotomy with resection of transverse colon and right thoracostomy, and another patient underwent a splenectomy.

Data on the earliest time to following commands were available for 203 patients with severe TBI and favorable outcomes at 6 months (Table 1). The majority of patients were able to follow commands (mGCS score of 6) within the 1st week after the injury (71.4\%), with $28.6 \%$ of patients recovering on Day 1 (Fig. 2). By Day 14 after the injury, $173(85.2 \%)$ patients were able to follow commands. The remaining patients began following commands by Day $30(12.3 \%)$ or Day 90 (2.5\%). Patients who began to follow commands later had significantly longer durations of ICP monitoring and neuromuscular blockade. There was no significant association between mass lesions on initial CT scans and time to following commands ( $\mathrm{p}=$ 0.37). Additionally, following commands on Day 1 after the injury was not significantly associated with mass le-

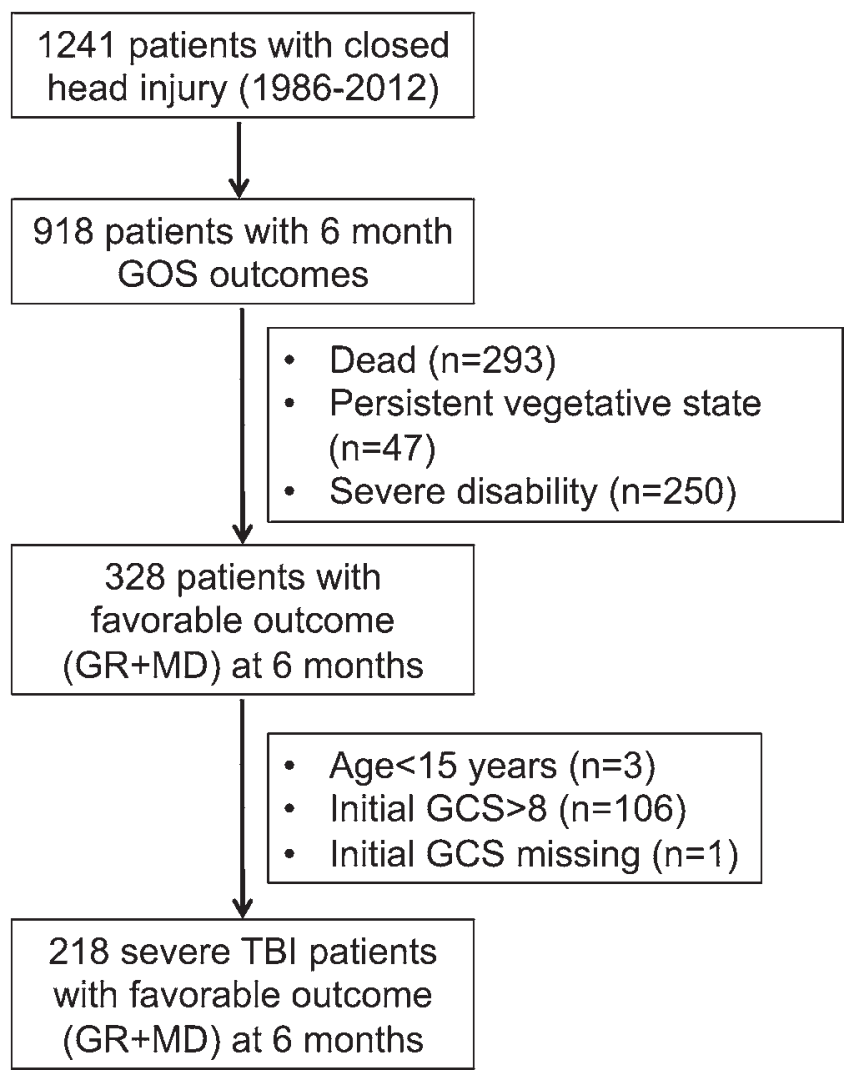

FIG. 1. Flow chart showing selection of patients with severe TBI and favorable outcomes at 6 months, from a research database of 1241 patients. $\mathrm{GR}=$ good recovery; MD = moderate disability. 
TABLE 1. Demographic, clinical, and imaging data categorized by time from injury to recovery to the point of following commands (mGCS score of 6) in 203 patients after severe TBI

\begin{tabular}{|c|c|c|c|c|c|}
\hline \multirow[b]{2}{*}{ Variable } & \multicolumn{5}{|c|}{ Time From Injury to 1st Record of Following Commands } \\
\hline & $\leq 7$ Days & 8-14 Days & 15-30 Days & 31-90 Days & p Value \\
\hline No. of pts & $145(71.4)$ & $28(13.8)$ & $25(12.3)$ & $5(2.5)$ & \\
\hline Age in yrs & $28.7 \pm 10.8$ & $32.4 \pm 13.2$ & $27.8 \pm 12.0$ & $22.6 \pm 5.7$ & 0.22 \\
\hline \multicolumn{6}{|l|}{ Sex } \\
\hline Male & $126(86.9)$ & $22(78.6)$ & $21(84)$ & $2(40)$ & \\
\hline Female & $19(13.1)$ & $6(21.4)$ & $4(16)$ & $3(60)$ & \\
\hline \multicolumn{6}{|l|}{ Mechanism of injury } \\
\hline MVA & $86(59.3)$ & $12(42.9)$ & $14(56)$ & $5(100)$ & 0.79 \\
\hline Assault & $17(11.7)$ & $6(21.4)$ & $4(16)$ & $0(0)$ & \\
\hline Fall/jump & $14(9.7)$ & $5(17.9)$ & $2(8)$ & $0(0)$ & \\
\hline Motorcycle & $14(9.7)$ & $2(7.1)$ & $4(16)$ & $0(0)$ & \\
\hline Other* & $14(9.7)$ & $3(10.7)$ & $1(4)$ & $0(0)$ & \\
\hline Prehospital hypoxia, $n=174$ & $33(19)$ & $8(4.6)$ & $7(4)$ & $2(1.1)$ & 0.62 \\
\hline Prehospital hypotension, $n=175$ & $9(5.1)$ & $3(1.7)$ & $3(1.7)$ & $1(0.6)$ & 0.22 \\
\hline Initial GCS score & $5.9 \pm 1.9$ & $6.0 \pm 1.8$ & $5.7 \pm 1.7$ & $6.2 \pm 1.3$ & 0.99 \\
\hline Initial mGCS score & $3.9 \pm 1.7$ & $3.8 \pm 1.9$ & $3.8 \pm 1.7$ & $4.0 \pm 1.2$ & 0.92 \\
\hline Initial pupil reactivity, $n=197$ & & & & & 0.50 \\
\hline Both reactive & $105(74.5)$ & $21(77.8)$ & $16(66.7)$ & $3(60)$ & \\
\hline Unilat unreactive & $12(8.5)$ & $0(0)$ & $3(12.5)$ & $0(0)$ & \\
\hline Bilat unreactive & $24(17)$ & $6(22.2)$ & $5(20.8)$ & $2(40)$ & \\
\hline Anisocoria & $26(18.4)$ & $3(11.1)$ & $7(29.2)$ & $1(20)$ & 0.39 \\
\hline ISS & $28.4 \pm 6.3$ & $28.4 \pm 6.4$ & $28.6 \pm 6.5$ & $35.6 \pm 9.3$ & 0.11 \\
\hline \multicolumn{6}{|l|}{ Initial Marshall CT class } \\
\hline $\mathrm{D} 1$ & $2(1.4)$ & $0(0)$ & $0(0)$ & $0(0)$ & \\
\hline $\mathrm{D} 2$ & $64(44.1)$ & $11(39.3)$ & $12(48)$ & $4(80)$ & \\
\hline D3 & $23(15.9)$ & $5(17.9)$ & $4(16)$ & $1(20)$ & \\
\hline D4 & $1(0.7)$ & $0(0)$ & $0(0)$ & $0(0)$ & \\
\hline M1 & $52(35.9)$ & $11(39.3)$ & $8(32)$ & $0(0)$ & \\
\hline M2 & $3(2.1 \%)$ & $0(0)$ & $1(4)$ & $0(0)$ & \\
\hline PBI & $0(0)$ & $1(3.6)$ & $0(0)$ & $0(0)$ & \\
\hline Surgical intervention, $n=74$ & $53(36.6)$ & $13(46.4)$ & $8(32)$ & $0(0)$ & 0.27 \\
\hline Time to surgery in hrs & $3.2 \pm 3.1$ & $2.7 \pm 1.9$ & $1.7 \pm 0.5$ & $0(0)$ & 0.34 \\
\hline Duration of ICP monitoring in hrs & $127.0 \pm 104.7(144 \mathrm{pts})$ & $178.9 \pm 101.2(28 \mathrm{pts})$ & $179.7 \pm 141.2(25 \mathrm{pts})$ & $271.9 \pm 74.0(5 \mathrm{pts})$ & 0.001 \\
\hline Duration of neuromuscular blockade in hrs & $104.8 \pm 91.4(138 \mathrm{pts})$ & $169.7 \pm 97.4(27 \mathrm{pts})$ & $158.9 \pm 148.7(24 \mathrm{pts})$ & $268.1 \pm 74.1$ (5 pts) & $<0.001$ \\
\hline Time to last dose of pentobarbital in hrs & $319.4 \pm 80.9$ (6 pts) & $170.6 \pm 90.1(5 \mathrm{pts})$ & $367.9 \pm 55.9$ (6 pts) & $241.5(1 \mathrm{pt})$ & ND \\
\hline LOS in ICU in days, $n=189$ & $15.1 \pm 8.6(136 \mathrm{pts})$ & $20.8 \pm 8.5(26 \mathrm{pts})$ & $24.4 \pm 9.1(22 \mathrm{pts})$ & $34.6 \pm 8.7$ (5 pts) & $<0.001$ \\
\hline LOS in hospital in days, $n=175$ & $22.7 \pm 14.6$ (125 pts) & $31.1 \pm 15.9(24 \mathrm{pts})$ & $32.9 \pm 13.7$ (21 pts) & $38.4 \pm 7.2(5 \mathrm{pts})$ & 0.001 \\
\hline
\end{tabular}

D1-4 = diffuse injury [Class I-IV]; LOS = length of stay; M1 = evacuated mass lesion [Class V]; M2 = nonevacuated mass lesion [Class VI]; MVA = motor vehicle accident; $\mathrm{ND}=$ not done; $\mathrm{PBI}=$ penetrating brain injury; pts = patients.

Unless otherwise indicated, values are reported as number (\%) or as the mean \pm SD. Boldface type indicates statistical significance.

* Includes the following: hit by falling object $(n=2)$, sports injury $(n=2)$, off-road vehicle crash $(n=3)$, bicycle crash $(n=2)$, commercial truck accident $(n=4)$, and unknown $(n=5)$.

sions $(\mathrm{p}=0.63)$ or primary decompressive surgery $(\mathrm{p}=$ $0.63)$.

Because the study period spans several years, we analyzed clinical factors and outcomes for each decade of the study: 1986-1995, 1996-2005, and 2006-2012. There was no significant association between years of injury and use of pentobarbital $(\mathrm{p}=0.13)$, decompressive surgery $(\mathrm{p}=$ $0.33)$, or GOS scores at 6 months $(\mathrm{p}=0.13)$. There was a significant decrease in the mean duration of ICU stay in the most recent cohort (1986-1995: $20.5 \pm 9.9$ days; $1996-$ 2005: $18.01 \pm 10.1$ days; and 2006-2012: $14.1 \pm 7.8$ days [ANOVA, $\mathrm{p}=0.001]$ ) but not in the duration of hospital stay (ANOVA, $\mathrm{p}=0.09$ ). Time to recovery to following commands did not vary significantly with years of injury (chi-square test, $\mathrm{p}=0.45$ ).

Patients with good recovery (GOS score of 5) had a 


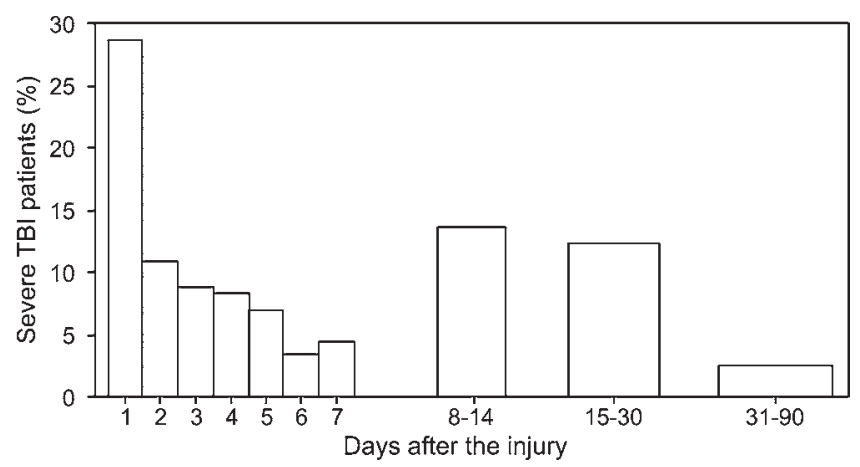

FIG. 2. Bar graph showing proportion of patients with severe TBI who recovered to an mGCS score of 6 at specific time points after the injury.

higher initial GCS score, lower incidence of anisocoria at admission, shorter ICU stay, and shorter total hospital stay (Table 2). Ninety-six of 218 patients (44\%) were discharged home. Other discharge dispositions included rehabilitation facility $(\mathrm{n}=81,37.2 \%)$, another hospital $(\mathrm{n}=$ $14,6.4 \%)$, long-term acute care facility $(\mathrm{n}=13,6 \%)$, and unspecified $(n=2,0.9 \%)$. Discharge disposition data were not available for 12 patients.

Of the 1241 patients, life-sustaining treatment was withdrawn in 40 individuals with severe TBI at our center. The median initial GCS score was 4/15, and 19 of these patients $(47.5 \%)$ presented with a GCS score of 3/15. Bilateral unreactive pupils were seen in 24 patients $(60 \%)$ in whom care was withdrawn. Twenty-five patients $(62.5 \%)$ underwent decompressive surgery, and 13 patients (32.5\%) underwent pentobarbital therapy. In comparison with patients with severe TBI who had favorable outcomes $(\mathrm{n}=$ 203), withdrawal of life-sustaining therapy was significantly associated with a GCS score of 3 at admission ( $p<$ $0.001)$, bilateral unreactive pupils at admission $(\mathrm{p}<0.001)$, and mass lesions on the initial CT scan for the Marshall grade $(\mathrm{p}=0.001)$.

The temporal profile of outcomes according to GOS scores from discharge to 6-month follow-up is shown in Fig. 3. The GOS scores at 6 months showed that the majority of patients had moderate disability $(n=115,52.8 \%)$. The correlation between 6-month GOS and 3-month GOS scores $(r=0.56, p<0.001)$ was stronger than that between 6-month GOS and 1-month GOS scores $(\mathrm{r}=0.33$, $\mathrm{p}<$ 0.001 ). There was no correlation between 6-month GOS and discharge GOS scores $(\mathrm{r}=0.02, \mathrm{p}=0.83)$.

\section{Discussion}

This study describes patients with favorable 6-month outcomes after severe TBI. The mean age of patients in this study was younger than 30 years; therefore, the results and interpretation of results are relevant to younger patients with severe TBI. All patients were treated by a neurointensivist and neurosurgeon, and the role of a dedicated neurocritical care team in guiding recovery is acknowledged.

We studied early recovery of gross motor function in patients as documented by the mGCS. Approximately $15 \%$ of patients began to follow commands beyond 2 weeks after the injury. Importantly, there were no significant dif-

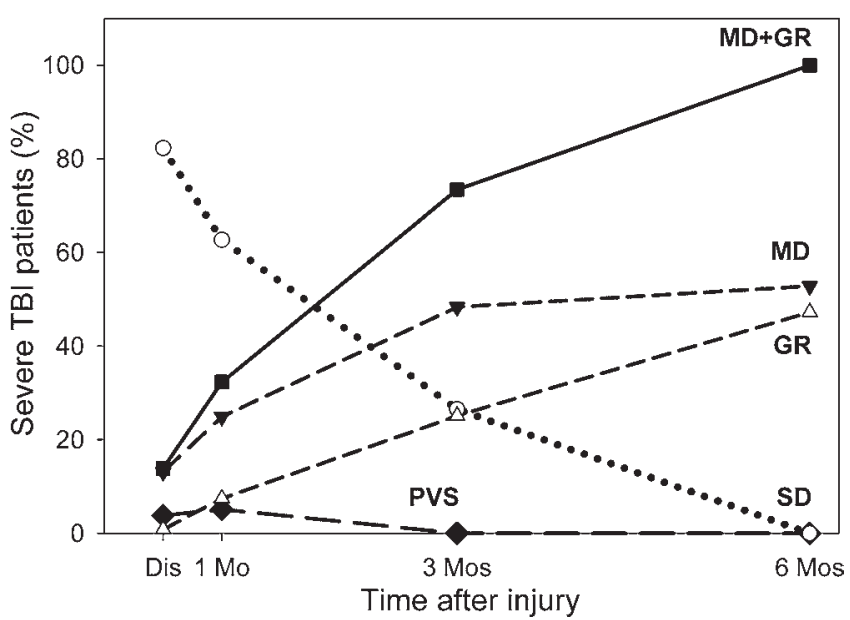

FIG. 3. Graph showing percentages for outcomes according to GOS scores from discharge to 1 month, 3 months, and 6 months after the injury in patients with favorable outcomes (moderate disability [MD] and good recovery [GR]) after severe TBI. Discharge outcome is presented for patients with a hospital stay of $<30$ days. Dis = discharge; PVS = persistent vegetative state; $\mathrm{SD}=$ severe disability.

ferences between the baseline clinical or imaging characteristics in this group of patients as compared with those who began to follow commands earlier. These patients, however, had undergone prolonged ICP monitoring and neuromuscular blockade, which may have impacted the early detection of motor recovery during the acute phase after severe TBI. It is therefore impossible to exclude a favorable outcome in patients with severe TBI who do not follow commands in the initial days after the injury. These findings highlight the challenges in accurately predicting a favorable outcome within the first 2 weeks after severe TBI, and cautions physicians against prematurely assuming a poor prognosis in patients with severe TBI who do not follow commands early after the injury. At our center, withdrawal of life-sustaining therapy was more likely in patients who had a severe initial injury characterized by a GCS score of 3/15 and bilaterally unreactive pupils at admission, both of which are known predictors of early mortality after TBI. ${ }^{12}$

Data from the present study provide valuable insights into the recovery potential and its expected timeline in patients with a favorable outcome after severe TBI. In the acute phase, it is often difficult to establish with certainty that a patient has no potential for a favorable outcome. Withdrawal of care is one of the most important contributors to in-hospital death after severe TBI. ${ }^{16}$ In some studies, life-sustaining care was withdrawn within 72 hours after the injury in patients with an initial mGCS score of $1-4 .^{6,16}$ In this study, the mean duration of ICP monitoring was approximately 6 days, and gross motor recovery (mGCS score of 6) was seen beyond 2 weeks in $15 \%$ of patients. Therefore, the timing and indication for withdrawal of care should be carefully considered, since early withdrawal of care may deprive some patients with severe TBI of a favorable outcome.

There are limited descriptions of the temporal profile of early recovery after severe TBI. We found improved functional outcomes in the first 6 months after the injury, and 
TABLE 2. Demographic, clinical, and imaging data for patients with favorable outcome (moderate disability and good recovery) at 6 months after severe TBI

\begin{tabular}{|c|c|c|c|c|}
\hline Variable & Total & Moderate Disability & Good Recovery & $\mathrm{p}$ Value \\
\hline No. of pts & 218 & 115 & 103 & \\
\hline Age in yrs & $28.9 \pm 11.2$ & $30.0 \pm 11.4$ & $27.7 \pm 10.9$ & 0.12 \\
\hline \multicolumn{5}{|l|}{ Sex } \\
\hline Male & $183(83.9)$ & $93(80.9)$ & $90(87.4)$ & 0.19 \\
\hline Female & $35(16.1)$ & $22(19.1)$ & $13(12.6)$ & \\
\hline \multicolumn{5}{|l|}{ Mechanism, $n=211$} \\
\hline MVA & $127(58.3)$ & $61(53)$ & $66(64.1)$ & 0.41 \\
\hline Assault & $29(13.3)$ & $18(15.7)$ & $11(10.7)$ & \\
\hline Fall/jump & $21(9.6)$ & $12(10.4)$ & $9(8.7)$ & \\
\hline Motorcycle & $20(9.2)$ & $10(8.7)$ & $10(9.7)$ & \\
\hline Other* & $21(9.6)$ & $14(12.2)$ & $7(6.8)$ & \\
\hline Prehospital hypoxia, n = 170 & $52(30.6)$ & $25(14.7)$ & $27(15.9)$ & 0.62 \\
\hline Prehospital hypotension, n = 171 & $19(11.1)$ & $12(7.0)$ & $7(4.1)$ & 0.65 \\
\hline Initial GCS score & $5.9 \pm 1.9$ & $5.6 \pm 1.8$ & $6.3 \pm 1.9$ & 0.01 \\
\hline Initial mGCS score & $3.9 \pm 1.9$ & $3.8 \pm 2.0$ & $4.0 \pm 1.9$ & 0.5 \\
\hline \multicolumn{5}{|l|}{ Initial pupil reactivity, $\mathrm{n}=211$} \\
\hline Both reactive & $155(73.5)$ & $77(69.4)$ & $78(78)$ & 0.15 \\
\hline Unilat unreactive & $16(7.6)$ & $12(10.8)$ & $4(4)$ & \\
\hline Bilat unreactive & $40(19)$ & $22(19.8)$ & $18(18)$ & \\
\hline Anisocoria & $37(17.5)$ & $25(22.5)$ & $12(12)$ & 0.048 \\
\hline ISS & $28.8 \pm 6.7$ & $28.4 \pm 6.2$ & $29.3 \pm 7.3$ & 0.3 \\
\hline \multicolumn{5}{|l|}{ Initial Marshall CT class } \\
\hline $\mathrm{D} 1$ & $2(0.9)$ & $1(0.9)$ & $1(1)$ & $0.57 \dagger$ \\
\hline $\mathrm{D} 2$ & $97(44.5)$ & $48(41.7)$ & $49(47.6)$ & \\
\hline D3 & $39(17.9)$ & $22(19.1)$ & $17(16.5)$ & \\
\hline D4 & $1(0.5)$ & $0(0)$ & $1(1)$ & \\
\hline M1 & $74(33.9)$ & $40(34.8)$ & $34(33)$ & \\
\hline M2 & $4(1.8)$ & $3(2.6)$ & $1(1)$ & \\
\hline PBI & $1(0.5)$ & $1(0.9)$ & $0(0)$ & \\
\hline Time to surgery in hrs & $2.9 \pm 2.7$ & $2.5 \pm 2.0$ & $3.2 \pm 3.4$ & 0.26 \\
\hline Duration of ICP monitoring in hrs & $143.5 \pm 111.5$ & $155.7 \pm 115.8$ & $129.5 \pm 105.2$ & 0.09 \\
\hline Duration of neuromuscular blockade in hrs & $121.8 \pm 104.6$ & $134.6 \pm 111.2$ & $107.5 \pm 95.2$ & 0.07 \\
\hline Time to last dose of pentobarbital in hrs & $295.1 \pm 106.8$ & $308.4 \pm 86.1$ & $272.34 \pm 140.3$ & 0.49 \\
\hline LOS in ICU in days, $n=199$ & $17.5 \pm 9.6$ & $19.9 \pm 9.9$ & $14.7 \pm 8.5$ & $<0.001$ \\
\hline LOS in hospital in days, $n=182$ & $26.0 \pm 15.4$ & $31.1 \pm 16.7$ & $20.1 \pm 11.2$ & $<0.001$ \\
\hline
\end{tabular}

this was more closely associated with the 3 -month GOS scores than with the GOS scores at discharge. Similar to results from prior studies, ${ }^{1,2,9}$ the patient's functional status when leaving the ICU or at discharge from the hospital was not consistently associated with outcome at follow-up. However, the correlation between 3-month and 6-month GOS scores was only moderate in the present study, and it is likely that other factors such as age and initial injury severity affect the neurological improvement between 3 and 6 months. ${ }^{2}$ Overall, in spite of a low initial GCS score being associated with increased morbidity, ${ }^{11,12,17}$ a propor- tion of patients with severe TBI do have the potential for considerable recovery and favorable outcomes.

A prognostic model to predict a favorable outcome after severe TBI would help neurointensivists better manage the care of these patients and offer families and physicians a guide to establishing goals of care. A prospective study of patients with severe TBI in whom care was not withdrawn would be required to develop a prognostic model for favorable outcomes. This would help determine the true outcome after severe TBI and identify baseline factors associated with favorable outcome. Multiple statisti- 
cal methods including regression analysis and recursive partitioning have been described to establish a prognostic model..$^{10}$ In the present study, we found that lower baseline GCS scores and anisocoria were associated with moderate disability and not with good recovery. Other studies have shown that age, mGCS score, pupil reactivity, type of intracranial lesion-traumatic subarachnoid hemorrhage or epidural hematoma-and the presence of major extracranial injury are associated with GOS scores at 6 months. ${ }^{12,15}$ These factors are likely to be contributors to a more accurate prognostic model for determining a favorable outcome in patients with severe TBI.

This study is limited by missing data for admission and follow-up variables at 1 and 3 months. Of the initial 1241 patients we did lose patients to attrition $(n=323)$, and this does contribute to bias in the study population. The small number of patients with a delay in following commands may have limited the ability to detect differences between groups, and this could be overcome by using larger multicenter data sets. The total duration of time spent on a ventilator, the duration of time that patients received sedation, and the occurrence of infections may have affected neurological recovery; however, we did not have data for these variables.

In the present study, CT imaging was used to establish baseline injury characteristics. We were able to compare recovery times for mass lesions and diffuse injury on initial CT, but not for individual pathologies such as epidural or subdural hematoma or contusion. Magnetic resonance imaging, which was not performed for this patient population, may help in better defining the extent of injury and may improve prognostication. ${ }^{18}$ Although the data span many years, the protocols for severe TBI management at our institution did not vary greatly, as reflected in our results, and the philosophy of avoiding early withdrawal of life-sustaining care for TBI was maintained throughout the study period. The lack of difference in the proportion of patients with delayed motor recovery from early to more recent years of the study suggests that recovery may be largely dependent on primary brain injury and initial injury severity, which makes prediction of motor recovery challenging for the individual patient. Overall, our study provides data to inform physicians and families on the expected time course for possible recovery to a favorable outcome after severe TBI.

\section{Conclusions}

In this study, up to $15 \%$ of patients began to follow commands beyond 2 weeks after the injury, indicating that a favorable outcome after severe TBI is possible even in patients with delayed motor recovery. Patients with favorable outcome after severe TBI showed marked improvement in GOS scores between discharge and follow-up at 6 months.

\section{References}

1. Braakman R, Jennett WB, Minderhoud JM: Prognosis of the posttraumatic vegetative state. Acta Neurochir (Wien) 95:49-52, 1988

2. Choi SC, Barnes TY, Bullock R, Germanson TA, Marmarou A, Young HF: Temporal profile of outcomes in severe head injury. J Neurosurg 81:169-173, 1994
3. Choi SC, Narayan RK, Anderson RL, Ward JD: Enhanced specificity of prognosis in severe head injury. J Neurosurg 69:381-385, 1988

4. Corral L, Ventura JL, Herrero JI, Monfort JL, Juncadella M, Gabarrós A, et al: Improvement in GOS and GOSE scores 6 and 12 months after severe traumatic brain injury. Brain Inj 21:1225-1231, 2007

5. Hukkelhoven CW, Steyerberg EW, Habbema JD, Farace E, Marmarou A, Murray GD, et al: Predicting outcome after traumatic brain injury: development and validation of a prognostic score based on admission characteristics. J Neurotrauma 22:1025-1039, 2005

6. Izzy S, Compton R, Carandang R, Hall W, Muehlschlegel S: Self-fulfilling prophecies through withdrawal of care: do they exist in traumatic brain injury, too? Neurocrit Care 19:347363,2013

7. Jennett B, Teasdale G, Braakman R, Minderhoud J, KnillJones R: Predicting outcome in individual patients after severe head injury. Lancet 1:1031-1034, 1976

8. Lang EW, Pitts LH, Damron SL, Rutledge R: Outcome after severe head injury: an analysis of prediction based upon comparison of neural network versus logistic regression analysis. Neurol Res 19:274-280, 1997

9. Levin HS, Saydjari C, Eisenberg HM, Foulkes M, Marshall LF, Ruff RM, et al: Vegetative state after closed-head injury. A Traumatic Coma Data Bank Report. Arch Neurol 48:580585,1991

10. Maas AI, Lingsma HF, Roozenbeek B: Predicting outcome after traumatic brain injury. Handb Clin Neurol 128:455474, 2015

11. Narayan RK, Greenberg RP, Miller JD, Enas GG, Choi SC, Kishore PR, et al: Improved confidence of outcome prediction in severe head injury. A comparative analysis of the clinical examination, multimodality evoked potentials, CT scanning, and intracranial pressure. J Neurosurg 54:751-762, 1981

12. Perel P, Arango M, Clayton T, Edwards P, Komolafe E, Poccock S, et al: Predicting outcome after traumatic brain injury: practical prognostic models based on large cohort of international patients. BMJ 336:425-429, 2008

13. Plaisier BR, Blostein PA, Hurt KJ, Malangoni MA: Withholding/withdrawal of life support in trauma patients: is there an age bias? Am Surg 68:159-162, 2002

14. Signorini DF, Andrews PJ, Jones PA, Wardlaw JM, Miller JD: Predicting survival using simple clinical variables: a case study in traumatic brain injury. J Neurol Neurosurg Psychiatry 66:20-25, 1999

15. Steyerberg EW, Mushkudiani N, Perel P, Butcher I, Lu J, McHugh GS, et al: Predicting outcome after traumatic brain injury: development and international validation of prognostic scores based on admission characteristics. PLoS Med 5:e165, 2008

16. Turgeon AF, Lauzier F, Simard JF, Scales DC, Burns KE, Moore L, et al: Mortality associated with withdrawal of life-sustaining therapy for patients with severe traumatic brain injury: a Canadian multicentre cohort study. CMAJ 183:1581-1588, 2011

17. Wagner AK, Hammond FM, Sasser HC, Wiercisiewski D, Norton HJ: Use of injury severity variables in determining disability and community integration after traumatic brain injury. J Trauma 49:411-419, 2000

18. Yuh EL, Mukherjee P, Lingsma HF, Yue JK, Ferguson AR, Gordon WA, et al: Magnetic resonance imaging improves 3 -month outcome prediction in mild traumatic brain injury. Ann Neurol 73:224-235, 2013

\section{Disclosures}

The authors report no conflict of interest concerning the materi- 
als or methods used in this study or the findings specified in this paper.

\section{Author Contributions}

Conception and design: Robertson. Acquisition of data: Vedantam, Robertson. Analysis and interpretation of data: all authors. Drafting the article: Gopinath, Vedantam. Critically revising the article: all authors. Reviewed submitted version of manuscript: all authors. Approved the final version of the manuscript on behalf of all authors: Gopinath. Statistical analysis: Vedantam. Administrative/technical/material support: Robertson. Study supervision: Gopinath, Robertson.

\section{Supplemental Information}

Previous Presentations

Portions of this work were submitted as an abstract for the American Association of Neurological Surgeons Annual Meeting, held in Los Angeles, CA, in 2017.

\section{Correspondence}

Shankar P. Gopinath, Department of Neurosurgery, Baylor College of Medicine, 7200 Cambridge, Ste. 9A, MS: BCM650, Houston, TX 77030. email: shankarg@bcm.edu. 